\title{
Eaux minérales et thermales en Pologne
}

\author{
J. Dowgiałło \\ Institut des Sciences Géologiques, Académie Polonaise des Sciences ( ${ }^{1}$ )
}

\section{GACTEURS PRINCIPAUX DÉCISIFS DE LA PRÉSENCE DES EAUX À VALEURS THÉRAPEUTIQUES}

La présence d’eaux souterraines qui peuvent être appliquées comme moyens thérapeutiques de thermalisme fut constatée sur presque tout le territoire de Pologne. Des exceptions regardent des régions limitées comme une partie mince du Nord-Est du pays ou certaines parts des Sudètes et de leur bloc précédent. Dans ces endroits le socle cristallin se trouve à la surface ou bien est couvert par une série sédimentaire peu épaisse. La zone d'échange intensif des eaux atteint ici les formations cristallines et si les conditions locales ne sont pas favorables à la formation des eaux spécifiques comme celles carbogazeuses, thermales ou radioactives, on ne trouve ici que des eaux simples, peu minéralisées (oligominérales). Ces eaux, selon les règlements polonais, ne peuvent pas être déclarées médicinales. Dans les autres régions on trouve les eaux minérales (contenant au moins $1000 \mathrm{mg} / \mathrm{l}$ de sels dissous avec souvent des composantes spécifiques thérapeutiques tels que le brome. l'iode. l'arsenic, etc., en quantités audessus des seuils pharmacodynamiques) $\left({ }^{2}\right)$.

(') ZWIRKI I WIGURY 93, 02-089 Warszawa, Pologne.

(2) Selon la loi, une eau peut être considérée comme curative si elle contient au moins $1000 \mathrm{mg} / \mathrm{l}$ de sels dissous (eaux minérales) ou des quantités précises des composantes ayant un effet curatif ou bien une température dépassant $20^{\circ} \mathrm{C}$ au griffon (eaux spécifiques) l'eau peut être déclarée curative seulement par le Ministre de la Santé.
Le fait que dans une région donnée apparaissent les eaux intéressantes du point de vue de la médecine balnéaire ne signifie pas qu'en n'importe quel point le captage et l'exploitation de ces eaux dans des quantités quelconques soient toujours possibles. Les possibilités d'extraction de ces eaux dépendent de la lithologie des couches aquifères ainsi que de la tectonique locale.

Aussi les profondeurs, dans lesquelles des eaux répondant aux exigences de la thérapie balnéaire peuvent être captées, varient dans un intervalle très large.

Parfois il y a des possibilités d'utilisation des sources naturelles et du captage de l'eau tout près de la surface. De telles sources étaient à l'origine du développement d'au moins une trentaine des stations thermales polonaises. Quelquefois, la profondeur sur laquelle on peut trouver une telle eau dépasse $1000 \mathrm{~m}$ et son captage exige des travaux de forage coûteux.

La répartition des types particuliers d'eaux souterraines qui peuvent trouver une application médicale est conforme, grosso modo, aux unités principales de la structure géologique de la Pologne. La région la plus riche en eaux thérapeutiques est celle des Sudètes avec leur bloc précédent.

On trouve une situation similaire dans les Carpates où, pourtant, dans la zone limitrophe avec leur fosse marginale il y a une zone de mélange des eaux du flysch des Carpates et de celles qui doivent leur composition chimique aux solutions connées marines du bassin miocène.

Les terrains situés au nord des Carpates dans lesquels la minéralisation des eaux souterraines est influencée par les sédiments du Miocène, sont difficiles à délimiter d'une manière exacte. C'est parce que dans les couches aquifères

\section{Mineral and thermal waters in Poland}

The curative waters used in Polish spas are formed from three main chemical types and their combinations: 1) saline water (sodium chloride) existing in the sedimentary deposits of the Northern Plain and in the Carpathians; 2) carbonated water in the Carpathians and the Sudetes region; 3) water containing hydrosulphides occurring primarily in the foreland of the Carpathians.

Various mineral waters are rich in elements such as iodine and bromine which have industrial applications; their extraction could become profitable. The high temperature of certain mineral waters is at the basis of a number of geothermal projects at various stages of advancement. 


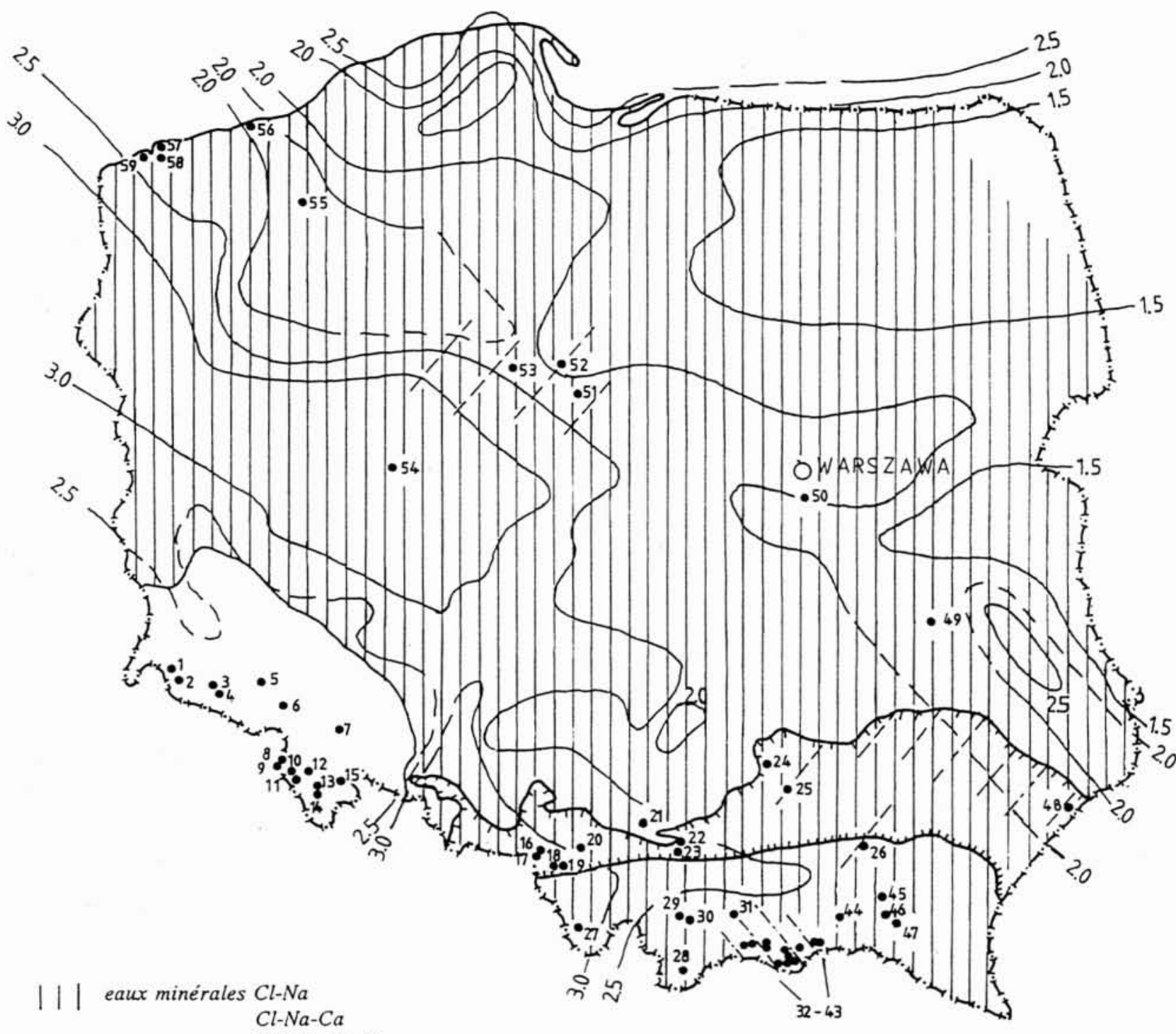

$\mathrm{Cl}-\mathrm{SO}_{4}-\mathrm{Na}-\mathrm{Ca}$

',', eaux à hydrosulfures

'! eaux carbogazeuses

$\therefore$ limite des Sudetes et de leur bloc précédent

limite des Carpathes

r. limite des formations marines du Miocène

3. - isolignes des valeurs moyennes du gradient de température $\left({ }^{\circ} \mathrm{C} / 100 \mathrm{~m}\right)$

- daprés Majorowicz, Plewa, 1979

2 - localités dont les eaux minérales et/ou spécifiques ont eté declarées curatives

\begin{tabular}{|c|c|c|c|}
\hline 1. Czerniawa & 16. Jastrzębie & 31. Szczawa & 46. Iwonicz \\
\hline 2. Swieradów & 17. Moszczenica & 32. Krościenko & 47. Rymanow \\
\hline 3. Cieplice & 18. Zabiocie & 33. Szczawnica & 48. Horyniec \\
\hline 4. Sosnowka & 19. Drogomysl & 34. Glębokie & 49. Nalęczow \\
\hline 5. Rochowice & 20. Goczalkowice & 35. Piwniczna & 50. Konstancin- \\
\hline 6. Szczawno & 21. Krzeszowice & 36. Komnica & olimów \\
\hline 7. Pizerzeczyn & 22. Kraków-Podgorze & 37. Zegiestow & 51. Wieniec \\
\hline 8. Kudowa & 23. Swoszowice & 38. Zlnckie & 52. Ciechocinek \\
\hline 9. Jeleniów & 24. Busiko & 39. Wierchomlâ & 53. Inowroclaw \\
\hline 10. Duszniki & 25. Solec & 40. Milik & 54. Koszuty \\
\hline 11. Bobrowniki Stare & 26. Latoszyn & 41. Muszyna & 55. Polczỵn \\
\hline 12. Polanica & 27. Sól & 42. Krynica & 56. Kolobrzeg \\
\hline 13. Gorzanów & 28. Zakopane & 43. Wysowa & 57. Dziwnotw \\
\hline 14. Dlugopole & 29. Rabika & 44. Wapienne & 58. Kamien Pomorski \\
\hline dek & 30. Poraba Wlk & 45. Krosno & 59. Swinoujscie \\
\hline
\end{tabular}

1. Les eaux minérales et thermales en Pologne : répartition régionale. 
profondes des formations du Mésozoïque inférieur et du Paléozoïque on trouve des eaux minérales qui du point de vue de leur genèse sont proches des eaux qu' on trouve dans le flysch des Carpates et de celles existant dans le souterrain des grands espaces de la Basse-Plaine du Nord. Ce sont des eaux chlorurées sodiques, parfois enrichies en Ca qui sont, en partie, des eaux connées des bassins sédimentaires marins. Du point de vue de l'origine des eaux minérales se trouvant dans les nappes profondes, les Carpates avec leur avant-fosse et la Basse Plaine feraient partie de la même zone.

Il s'ensuit que la présence de types particuliers d'eaux minérales ou spécifiques n'est pas, d'une manière très stricte, liée aux régions géologiques et des eaux typiques de certaines régions peuvent aussi être trouvées dans d'autres. Cela ne concerne pas seulement la composition chimique des eaux mais aussi leur température. Les circonstances particulièrement favorables à la formation et à l'émergence d'eaux thermales existent, dans les régions de montagne où les dénivellations du terrain permettent aux eaux de précipitation atmosphérique de pénétrer à des profondeurs considérables, d'acquérir des températures élevées et d'émerger dans les vallées situées en dessous de la zone dalimentation. Des circonstances de ce genre existent dans les Tatras (Carpates intérieures) et dans quelques parties des Sudètes, également dans quelques zones de la Basse-Plaine où il existe des conditions favorables à la présence d'eaux thermales. II s'agit bien sûr des eaux qui existent aux profondeurs économiquement raisonnables (des centaines et pas des milliers de mètres) et d'une minéralisation assez faible pour qu'elles puissent être appliquées sans nécessité de dilution. Cela concerne la partie sud-ouest de la Basse-Plaine où, d'un côté, on observe des valeurs relativement élevées du gradient géothermique, tandis que de l'autre côté, les nappes aquifères de Mésozoïque sont bien perméables et leurs zones d'alimentation (affleurements à la surface sous-Kénozoïque) ne sont pas très éloignées comme, par exemple, sur la monocline avant-Sudètes. De telles eaux ont été trouvées dans un forage à Koszuty où la température de l'eau s'écoulant spontanément du forage est de $42{ }^{\circ} \mathrm{C}$ tandis que sa minéralisation ne dépasse pas $9 \mathrm{~g} / \mathrm{l}$. Des paramètres si avantageux de l'eau ne sont pas cependant une généralité dans la Basse-Plaine. Même si le degré géothermique est bas et que la couche aquifere se caractérise par une perméabilité considérable, la minéralisation augmentant avec la profondeur disqualifie souvent l'eau qui ne peut pas être appliquée directement. La nécessité de la diluer exige des installations supplémentaires, ce qui met en doute la rentabilité d'exploitation de l'eau. En plus, on se heurte à l'aspect écologique de l'évacuation des eaux usées. La salinité exclue leur rejet dans les cours d'eau de surface et leur foulage dans les couches aquifères pose souvent des problèmes techniques et économiques considérables.

Malgré les difficultés mentionnées ci-dessus, en ce qui concerne la distinction des régions des eaux applicables à la médecine thermale, on adopte en général le principe de la division de la Pologne en quatre régions, en se basant sur les éléments principaux de la géologie du pays. Ce sont: les Carpates, le domaine de la présence des sédiments marins chimiques du Miocène, les Sudètes avec leur bloc précédent et la Basse-Plaine. On trouvera ci-dessous une caractérisation concise des régions particulières. Quelques détails peuvent être aussi trouvés dans le tableau ci-joint.

\section{DES CARPATES}

La partie la plus étendue des eaux minérales exploitées dans la région des Carpates se trouve dans les formations du flysch d’âge Tertiaire et Crétacé. Les plus répandues sont ici les eaux chlorurées sodiques à minéralisation entre quelques g/l jusqu'à quelques dizaines de $\mathrm{g} / \mathrm{l}$ et parfois plus que $100 \mathrm{~g} / \mathrm{l}$. En général on les trouve dans les forages à profondeurs différentes, parfois elles paraissent aussi à la surface comme sources. De telles sources existaient autrefois à Rabka où elles alimentaient une saunerie, à Sól près de Żywiec et dans quelques autres localités.

Les eaux chlorurées sodiques sont considérées comme polygéniques, contenant une composante connée des bassins marins. Cette hypothèse est confirmée par leur haute teneur en isotopes lourds d'oxygène et d'hydrogène. Elles contiennent souvent des quantités élevées de brome et d'iode. La concentration de ces ions, surtout d'iode, est liée à la présence dans les sédiments « flyscheux » de la matière organique, en particulier des hydrocarbures. Dans plusieurs cas on observe dans ces eaux la présence de quantités considérables de méthane (par exemple à Rabka, à Poręba Wielka, etc.). Parfois des processus d'oxydation des hydrocarbures conduisent à la génération de gaz carbonique Ce gaz apparaît alors dans les eaux minérales, contribuant à la formation des eaux chlorurées-bicarbonatées-sodiques $\left(\mathrm{Cl}-\mathrm{HCO}_{3}-\mathrm{Na}\right)$ qu'on trouve par exemple à Iwonicz et Rymanów.

Comparée à la présence commune des eaux chlorurées sodiques, la zone des Carpates dans laquelle les eaux carbogazeuses apparaissent, est mince et limitée surtout aux bassins des rivières Poprad et Dunajec. Le gaz carbonique étant la composante la plus caractéristique de ces eaux, est en général d'origine profonde. Il est probablement lié à l'ascension du magma basique qui, dans le Tertiaire supérieur, a causé l'activité volcanique dans la chaîne des Klippes de Pieniny et dans le Vihorlat en Slovaquie. Migrant vers la surface, ce gaz sature les eaux souterraines, exerçant une influence considérable sur leur composition chimique.

Résultant du contact du $\mathrm{CO}_{2}$ avec des eaux peu profondes d'origine atmosphérique se forment les eaux carbogazeuses bicarbonatées-calciques $\left(\mathrm{HCO}_{3}-\mathrm{Ca}\right)$ bicarbonatées-magnésiennes $\left(\mathrm{HCO}_{3}-\mathrm{Mg}\right)$ ou bien les combinaisons de ces deux types $\left(\mathrm{HCO}_{3}-\mathrm{Ca}-\mathrm{Mg}\right)$ avec une minéralisation ne dépassant pas quelques grammes par litre. Parmi les localités dans lesquelles ces eaux émergent à la surface, on peut nommer Krynica, Muszyna, Żegiestów, Milik, Wierchomla, Łomnica, Piwniczna et Głębokie.

Un autre phénomène plus compliqué est à l'origine d'eaux dans lesquelles outre la composante d'infiltration atmosphérique, on trouve une addition d'eaux connées. mentionnées ci-dessus, du type chloruré-sodique ( $\mathrm{Cl}-\mathrm{Na})$. Le résultat de saturation de telles eaux en gaz carbonique est la formation des eaux carbogazeuses bicarbonatéeschlorurées-sodiques $\left(\mathrm{HCO}_{3}-\mathrm{Cl}-\mathrm{Na}\right)$ souvent contenant des quantités élevées d'iode et de brome. Leur minéralisation est comprise entre quelques grammes par litre et quelques dizaines de $\mathrm{g} / \mathrm{l}$. On les trouve à la périphérie de la zone des eaux carbogazeuses des Carpates. Dans le nord et l'ouest de cette zone, les sources de ces eaux se trouvent à Szczawnica, Krościenko et Szczawa, dans l'est - à Wysowa. 
Tableau 1. - Liste des données concernant les eaux déclarées curatives.

\begin{tabular}{|c|c|c|c|c|c|c|c|}
\hline \multirow{2}{*}{ Localité } & \multirow{2}{*}{$\begin{array}{l}\text { Altitude } \\
\text { (m) }\end{array}$} & \multicolumn{2}{|c|}{ Captages } & \multirow{2}{*}{ Age des nappes } & \multirow{2}{*}{ Types d'eaux } & \multirow{2}{*}{$\begin{array}{c}\text { Minéralisation } \\
(\mathrm{g} / \mathrm{l})\end{array}$} & \multirow{2}{*}{$\begin{array}{c}\text { Température } \\
\left({ }^{\circ} \mathrm{C}\right)\end{array}$} \\
\hline & & Nombre & $\begin{array}{c}\text { Profondeur } \\
(\mathrm{m})\end{array}$ & & & & \\
\hline \multicolumn{8}{|c|}{ Les Carpates } \\
\hline Zakopane & $840^{*}$ & 1 & 1560 & Trias & $\mathrm{H}_{2} \mathrm{~S}$ & 0,35 & 37 \\
\hline Sól & $585^{*}$ & 1 & 1300 & $\begin{array}{l}\text { Tertiaire, } \\
\text { Crétacé }\end{array}$ & $\mathrm{Cl}-\mathrm{Na}$ & 42,6 & 35 \\
\hline Rabka & $510^{*}$ & 9 & $19-460$ & Tertiaire & $\mathrm{Cl}-\mathrm{Na}+\mathrm{J}+\mathrm{Br}$ & $8-25$ & 27 \\
\hline $\begin{array}{l}\text { Poręba } \\
\text { Wielka }\end{array}$ & $530^{*}$ & 1 & 1600 & - & $\mathrm{Cl}-\mathrm{Na}+\mathrm{J}+\mathrm{Br}$ & 21,5 & \\
\hline Szczawa & $520^{*}$ & 5 & $2-8$ & - & $\begin{array}{l}\mathrm{HCO}_{3}-\mathrm{Cl}-\mathrm{Na}+\mathrm{Br}+\mathrm{J}+ \\
\mathrm{CO}_{2}\end{array}$ & $1-7$ & \\
\hline Krościenko & 420 & 2 & $2-25$ & - & $\mathrm{HCO}_{3}-\mathrm{Cl}-\mathrm{Na}+\mathrm{J}+\mathrm{CO}_{2}$ & $8-11$ & \\
\hline Szczawnica & $480^{*}$ & 10 & 2 & $\begin{array}{l}\text { Tertiaire, } \\
\text { Crétacé }\end{array}$ & $\mathrm{HCO}_{3}-\mathrm{Cl}-\mathrm{Na}+\mathrm{J}+\mathrm{CO}_{2}$ & $3-13$ & \\
\hline Piwniczna & $400^{\circ}$ & 2 & $122-177$ & Tertiaire & $\mathrm{HCO}_{3}-\mathrm{Ca}-\mathrm{Mg}+\mathrm{CO}_{2}$ & 4 & \\
\hline Głębokie & 380 & 1 & 2 & - & $\mathrm{HCO}_{3}-\mathrm{Na}-\mathrm{Ca}+\mathrm{CO}_{2}$ & 3 & \\
\hline Łomnica & $475^{*}$ & 2 & 2 & - & $\mathrm{HCO}_{3}-\mathrm{Ca}+\mathrm{J}+\mathrm{CO}_{2}$ & $1,5-25$ & \\
\hline Wierchomla & $500^{*}$ & - & - & - & $\mathrm{HCO}_{3}-\mathrm{Ca}+\mathrm{J}+\mathrm{CO}_{2}$ & 2 & \\
\hline Żegiestów & $480^{*}$ & 4 & $2-300$ & - & $\mathrm{HCO}_{3}-\mathrm{Ca}-\mathrm{Mg}+\mathrm{CO}_{2}$ & $2,4-7,2$ & \\
\hline Milik & 485 & - & - & - & $\mathrm{HCO}_{3}-\mathrm{Ca}+\mathrm{CO}_{2}$ & 1 & \\
\hline Muszyna & 460 & 8 & $2-146$ & - & $\mathrm{HCO}_{3}-\mathrm{Ca}-\mathrm{Mg}+\mathrm{CO}_{2}$ & $1,2-8,1$ & \\
\hline Krynica & $560^{*}$ & 20 & $2-936$ & - & $\begin{array}{l}\mathrm{HCO}_{3}-\mathrm{Ca}+\mathrm{CO}_{2} \\
\mathrm{HCO}_{3}-\mathrm{Ca}-\mathrm{Na}-\mathrm{Mg}+\mathrm{CO}_{2} \\
\mathrm{HCO}_{3}-\mathrm{Na}+\mathrm{J}+\mathrm{CO}_{2}\end{array}$ & $\begin{array}{c}0,8-3 \\
2,4-5,1 \\
22,4-28,7\end{array}$ & \\
\hline Wysowa & 510 & 8 & $2-100$ & Crétacé & $\begin{array}{l}\mathrm{HCO}_{3}-\mathrm{Cl}-\mathrm{Na}+\mathrm{J}+\mathrm{Br}+ \\
\mathrm{CO}_{2}+ \\
\mathrm{HCO}_{3}-\mathrm{Na}-\mathrm{Ca}+\mathrm{CO}_{2} \\
\mathrm{HCO}_{3}-\mathrm{Cl}-\mathrm{Na}+\mathrm{J}+\mathrm{CO}_{2}\end{array}$ & $\begin{array}{c}23,6 \\
9,8 \\
1,6-5,7\end{array}$ & \\
\hline Wapienne & 383 & 2 & $2-5$ & Crétacé & $\mathrm{H}_{2} \mathrm{~S}$ & 0,4 & \\
\hline Krosno & 250 & 2 & $530-630$ & Tertiaire & $\mathrm{Cl}-\mathrm{Na}+\mathrm{Br}+\mathrm{J}$ & $26-43$ & \\
\hline Iwonicz & 410 & 5 & $231-985$ & - & $\mathrm{Cl}-\mathrm{HCO}_{3}-\mathrm{Na}+\mathrm{Br}+\mathrm{J}$ & $8-18$ & \\
\hline Rymanów & 400 & 4 & $2-470$ & - & $\mathrm{Cl}-\mathrm{HCO}_{3}-\mathrm{Na}+\mathrm{Br}+\mathrm{J}$ & $8,6-15$ & \\
\hline \multicolumn{8}{|c|}{ Le domaine des sédiments marins du Miocène } \\
\hline Jastrzębie & 280 & 1 & 350 & Carbonifère & $\mathrm{Cl}-\mathrm{Na}+\mathrm{Br}+\mathrm{J}$ & 17 & \\
\hline Moszczenica & 270 & 1 & 506 & - & $\mathrm{Cl}-\mathrm{Na}+\mathrm{Br}+\mathrm{J}$ & 28 & \\
\hline Drogomyśl & 260 & 1 & 691 & - & $\mathrm{Cl}-\mathrm{Na}+\mathrm{Br}+\mathrm{J}$ & 40 & \\
\hline Dębowiec & 280 & 4 & $400-500$ & Miocène & $\mathrm{Cl}-\mathrm{Na}+\mathrm{Br}+\mathrm{J}$ & $28-55$ & \\
\hline Zabłocie & 257 & 2 & $650-700$ & - & $\mathrm{Cl}-\mathrm{Na}+\mathrm{Br}+\mathrm{J}$ & 42 & \\
\hline Goczałkowice & 255 & 3 & $320-600$ & Carbonifère & $\mathrm{Cl}-\mathrm{Na}+\mathrm{Br}+\mathrm{J}$ & $62-72$ & \\
\hline Krzeszowice & 266 & 3 & $2-3$ & Miocène & $\mathrm{SO}_{4}-\mathrm{Ca}-\mathrm{Mg}+\mathrm{H}_{2} \mathrm{~S}$ & $2-4$ & \\
\hline Swoszowice & 250 & 2 & $1-10$ & 一 & $\mathrm{SO}_{4}-\mathrm{HCO}_{3}-\mathrm{Ca}-\mathrm{Mg}+\mathrm{H}_{2} \mathrm{~S}$ & $0,7-2,5$ & \\
\hline Kraków-Podgórze & 220 & 1 & 32 & - & $\mathrm{SO}_{4}-\mathrm{Cl}-\mathrm{Na}-\mathrm{Mg}-\mathrm{Ca}+\mathrm{H}_{2} \mathrm{~S}$ & 2,9 & \\
\hline
\end{tabular}


EAUX MINÉRALES ET THERMALES EN POLOGNE

\begin{tabular}{|c|c|c|c|c|c|c|c|}
\hline \multirow[b]{2}{*}{ Localité } & \multirow{2}{*}{$\begin{array}{c}\text { Altitude } \\
(\mathrm{m})\end{array}$} & \multicolumn{2}{|c|}{ Captages } & \multirow[b]{2}{*}{ Age des nappes } & \multirow[b]{2}{*}{ Types d'eaux } & \multirow{2}{*}{$\begin{array}{l}\text { Minéralisation } \\
(\mathrm{g} / \mathrm{l})\end{array}$} & \multirow{2}{*}{$\begin{array}{l}\text { Température } \\
\left({ }^{\circ} \mathrm{C}\right)\end{array}$} \\
\hline & & Nombre & \begin{tabular}{|} 
Profondeur \\
$(\mathrm{m})$
\end{tabular} & & & & \\
\hline Busko & 215 & 6 & $50-500$ & $\begin{array}{l}\text { Crétacé, } \\
\text { Jurassique }\end{array}$ & $\begin{array}{l}\mathrm{Cl}-\mathrm{Na}+\mathrm{H}_{2} \mathrm{~S} \\
\mathrm{Cl}-\mathrm{Na}+\mathrm{Br}+\mathrm{J}\end{array}$ & $\begin{array}{c}12-14 \\
23\end{array}$ & \\
\hline Solec & 300 & 2 & $120-140$ & Crétacé & $\mathrm{Cl}-\mathrm{Na}+\mathrm{J}+\mathrm{H}_{2} \mathrm{~S}$ & 20 & \\
\hline Latoszyn & 234 & 1 & 6 & Miocène & $\mathrm{SO}_{4}-\mathrm{Cl}-\mathrm{Na}+\mathrm{H}_{2} \mathrm{~S}$ & 5 & \\
\hline Horyniec & 250 & 1 & 20 & - & $\mathrm{H}_{2} \mathrm{~S}$ & 0,6 & \\
\hline \multicolumn{8}{|c|}{ Les Sudètes et leur bloc précédent } \\
\hline Lądek & $450^{*}$ & 5 & $2-5$ & Précambrien & $\mathrm{Rn}+\mathrm{F}+\mathrm{HS}^{-}$ & 0,2 & $24-24$ \\
\hline Długopole & 370 & 4 & $2-10$ & - & $\mathrm{HCO}_{3}-\mathrm{Ca}-\mathrm{Mg}-\mathrm{Na}+\mathrm{CO}_{2}$ & $1-2$ & \\
\hline Gorzanów & 313 & 4 & $160-280$ & Crétacé & $\mathrm{HCO}_{3}-\mathrm{Ca}-\mathrm{Na}+\mathrm{CO}_{2}$ & $1,2-1,7$ & \\
\hline Polanica & $367^{*}$ & 3 & $34-270$ & - & $\mathrm{HCO}_{3}-\mathrm{Ca}+\mathrm{CO}_{2}$ & $1,5-2,6$ & \\
\hline $\begin{array}{l}\text { Bobrowniki } \\
\text { Stare }\end{array}$ & 510 & - & - & Précambrien & $\mathrm{HCO}_{3}-\mathrm{Ca}-\mathrm{Mg}+\mathrm{CO}_{2}$ & 0,9 & \\
\hline Duszniki & 568 & 4 & $34-160$ & - & $\mathrm{HCO}_{3}-\mathrm{Ca}-\mathrm{Mg}+\mathrm{CO}_{2}$ & $1-3$ & \\
\hline Kudowa & 400 & 6 & $2-10$ & Quaternaire & $\mathrm{HCO}_{3}-\mathrm{Ca}-\mathrm{Na}+\mathrm{As}+\mathrm{CO}_{2}$ & $1,5-5$ & \\
\hline Przerzeczyn & 242 & 1 & 3 & - & $\mathrm{H}_{2} \mathrm{~S}$ & 0,4 & \\
\hline Szczawno & 410 & 9 & $1-3$ & Carbonifère & $\mathrm{HCO}_{3}-\mathrm{Na}-\mathrm{Ca}+\mathrm{CO}_{2}$ & $1,2-6$ & \\
\hline $\begin{array}{l}\text { Rochowice } \\
\text { Stare }\end{array}$ & 357 & 5 & $3-80$ & $\begin{array}{l}\text { Cambrien, } \\
\text { Ordovicien }\end{array}$ & $\mathrm{HCO}_{3}-\mathrm{SO}_{4}-\mathrm{Ca}-\mathrm{Na}-\mathrm{Mg}+\mathrm{CO}_{2}$ & $0,3-3,3$ & \\
\hline Sosnówka & 740 & 1 & 2 & Carbonifère & Rn & 0,2 & \\
\hline Cieplice & 347 & 6 & $2-166$ & - & $\mathrm{F}+\mathrm{H}_{2} \mathrm{SiO}_{3}$ & $0,5-0,7$ & $22-43,5$ \\
\hline Śweradów & 500 & 12 & $2-15$ & Précambrien & $\mathrm{HCO}_{3}-\mathrm{Ca}-\mathrm{Mg}+\mathrm{CO}_{2}$ & $\begin{array}{l}0,1 \\
1-2\end{array}$ & \\
\hline Czerniawa & 540 & 3 & $35-90$ & - & $\mathrm{HCO}_{3}-\mathrm{Ca}-\mathrm{Mg}+\mathrm{CO}_{2}$ & $1-2$ & \\
\hline \multicolumn{8}{|c|}{ La Basse-Plaine } \\
\hline Nałęczów & 170 & 3 & $2-4$ & Crétacé & $\mathrm{Fe}$. & 0,7 & \\
\hline Konstancin & 92 & 1 & 1740 & Jurassique & $\mathrm{Cl}-\mathrm{Na}+\mathrm{Br}+\mathrm{J}$ & 67,5 & 30 \\
\hline Wieniec & 64 & 1 & 131 & - & $\mathrm{SO}_{4}-\mathrm{Cl}-\mathrm{Ca}-\mathrm{Na}+\mathrm{Br}+\mathrm{H}_{2} \mathrm{~S}$ & 3,5 & \\
\hline Koszuty & 76 & 1 & 1020 & - & $\mathrm{Cl}-\mathrm{Na}$ & 8 & 41,8 \\
\hline Inowractaw & 87 & 1 & 3 & Quaternaire & $\mathrm{Cl}-\mathrm{Na}$ & 8,5 & \\
\hline Ciechocinek & 36 & 6 & $24-1450$ & $\begin{array}{l}\text { Quaternaire, } \\
\text { Jurassique }\end{array}$ & $\begin{array}{l}\mathrm{Cl}-\mathrm{Na} \\
\mathrm{Cl}-\mathrm{Na}+\mathrm{Br}+\mathrm{J}+\mathrm{H}_{2} \mathrm{~S}\end{array}$ & $\begin{array}{c}4-7 \\
42-70\end{array}$ & $27-37$ \\
\hline Połczyn & 115 & 1 & 1235 & Trias & $\mathrm{Cl}-\mathrm{Na}+\mathrm{Br}+\mathrm{J}$ & 74 & \\
\hline Świnoujście & 4 & 2 & $185-260$ & Crétacé & $\mathrm{Cl}-\mathrm{Na}+\mathrm{Br}+\mathrm{J}$ & $35-45$ & \\
\hline Dziwnów & 4 & 3 & $140-190$ & Jurassique & $\mathrm{Cl}-\mathrm{Na}+\mathrm{Br}$ & $30-40$ & \\
\hline $\begin{array}{l}\text { Kamień } \\
\text { Pomorski }\end{array}$ & 6 & 1 & 415 & - & $\mathrm{Cl}-\mathrm{Na}+\mathrm{Br}+\mathrm{J}$ & 38 & \\
\hline Kołobrzeg & 5 & 5 & $40-203$ & Quaternaire & $\mathrm{Cl}-\mathrm{Na}+\mathrm{Br}$ & $2-59$ & \\
\hline
\end{tabular}

1. La température des eaux dont les valeurs ne sont pas indiquées est inférieure à $20^{\circ} \mathrm{C}$.

2. signifie que les différences d'altitude dans le lieu donné ne dépassent pas $100 \mathrm{~m}$. 
Le bioxyde de carbone présent dans les eaux facilite le passage en solution des éléments présents dans les minéraux des roches aquifères. Selon le type de ces roches, les eaux carbogazeuses d'origine météorique se caractérisent par la prépondérance parmi les cations du calcium, du magnésium et parfois du sodium. Un enrichissement exceptionnellement haut en sodium se trouve parfois dans des eaux se trouvant à profondeurs considérables. En dehors du sodium, qui est une composante normale des eaux connées des bassins marins il y a ici du sodium provenant de la décomposition en présence de $\mathrm{CO}_{2}$ des minéraux constituants des roches. Ce sont surtout les feldspaths sodiques qui se trouvent en abondance dans les grès arkosiques du flysch. L'exemple classique de telles eaux carbogazeuses, bicarbonatées - sodiques sont les eaux «Zuber» de Krynica qu'on trouve dans les forages à des profondeurs dépassant $600 \mathrm{~m}$. Leur minéralisation dépasse $20 \mathrm{~g} / \mathrm{l}$, leur composante spécifique est souvent l'iode. D'après les recherches récentes, des eaux de ce type peuvent être trouvées dans beaucoup d'autres localités. Presque dans toute la zone des eaux carbogazeuses des Carpates, on observe l'augmentation de la teneur en sodium et de la minéralisation avec la profondeur. Il en résulte que la découverte des eaux de type «Zuber » dépend en quelque sorte de la profondeur de forage.

\section{LE DOMAINE DES SÉDIMENTS DU MIOCÈNE MARIN}

Les sédiments de la mer du Miocène occupent les territoires au nord des Carpates. Ils sont surtout répandus dans le bassin de Sandomierz sur la Vistule. Vers l'ouest, dans la région de Cracovie, leur étendue diminue et s'élargit de nouveau dans la Haute-Silésie. Par endroits, les sédiments du Miocène dépassent vers le sud le bord des Carpates. Ils couvrent là-bas les formations du flysch ou bien sont plissés avec eux.

Du point de vue de la formation des eaux applicables au thermalisme, les séries gypsifères du Miocène supérieur (Tortonien) sont d'une importance particulière. L'hydrogène sulfuré généré après la réduction bactérienne des gypses, se dissout dans les eaux d'infiltration simples et ainsi se forment les eaux simples à hydrosulfures (Horyniec, Latoszyn). Parfois l'hydrogène sulfuré est en contact avec des eaux qui sont génétiquement liées au Miocène ou bien aux formations plus anciennes. Dans ces cas-là se forment les eaux minérales chlorurées-sodiques à hydrosulfures ( $\mathrm{Cl}-\mathrm{Na}+\mathrm{H}_{2} \mathrm{~S}$ ) contenant souvent aussi l'iode en quantités dépassant $1 \mathrm{mg} / \mathrm{l}$ (Busko, Solec). Si la minéralisation des eaux est causée surtout par le lessivage des gypses, on trouve des eaux sulfatées-calciques, à hydrosulfures comme celles de Krzeszowice et Swoszowice. Un exemple d'eaux à hydrosulfures formées par le lessivage de gypse et contenant l'ingrédient chloruré-sodique, sont les eaux utilisées dans l'établissement thermal « Mateczny " à Kraków.

Sur tout le territoire couvert par des sédiments marins du Miocène supérieur, on trouve dans les nappes plus profondes de Mésozoïque les eaux chlorurées-sodiques enrichies en brome et iode. Leur minéralisation augmente avec la profondeur. A Busko une eau d'un tel type chimique est extraite à la profondeur de $500 \mathrm{~m}$ des calcaires du Jurassique supérieur. Sa minéralisation ( $23 \mathrm{~g} / \mathrm{l})$ dépasse presque deux fois celle des eaux chlorurées-sodiques, à hydrosulfures, qu'on trouve dans les formations de Crétacé.

Dans la Haute-Silésie où les sédiments de Miocène sont aussi présents, il manque des eaux à hydrosulfures. Dans les couches du Miocène ainsi que du Carbonifère on y trouve des eaux chlorurées-sodiques souvent considérablement enrichies en iode (jusqu'à $140 \mathrm{mg} / \mathrm{l}$ à Drogomyśl et Dębowiec). Ces eaux contiennent aussi du brome (parfois jusqu'à $200 \mathrm{mg} / \mathrm{l}$ ) à composition isotopique d'oxygène et d'hydrogène, qui permet de les définir comme eaux connées marines presque intactes. Les eaux de ces types ont été déclarées comme curatives à Jastrzębie, Moszczenica, Drogomyśl, Dẹbowiec, Zabłocie et Goczałkowice.

\section{IV — SUDÈTES ET LEUR BLOC PRÉCÉ- DENT}

Contrairement aux régions décrites ci-dessus, la présence des eaux minérales est limitée ici à quelques zones au-delà desquelles ces eaux n'apparaissent pas. C'est le résultat de l'absence sur des terrains étendus de cette région, de séries épaisses sédimentaires marines contenant des eaux connées ayant, en général, une minéralisation élevée. Aussi les dénivellations considérables des terrains de montagne et un réseau développé des fractures tectoniques facilitent-ils une circulation intense des eaux et ne favorisent pas l'augmentation de leur minéralisation à la suite d'un contact prolongé avec les roches. Eaux thermales et radioactives peuvent être trouvées en dehors des lieux où elles sont déjà connues. Cela concerne surtout des terrains granitiques et gneissiques.

Le facteur qui contribue à la formation des eaux minérales dans la région des Sudètes est le bioxyde de carbone se dégageant des parties profondes de l'écorce ou même du manteau terrestre, dans les zones des perturbations tectoniques particulières. Comme dans les Carpates il peut provenir directement du magma ou bien se produire par la décomposition thermique des roches calcaires. La présence de ces dernières n'est pas rare dans les formations métamorphiques des Sudètes. Les régions dans lesquelles la présence de $\mathrm{CO}_{2}$ entraîne la formation d'eaux carbogazeuses, sont le Bassin de Kłodzko (Kudowa, Polanica, Duszniki, Długopole, Gorzanów, Bobrowniki St.), la région des Sudètes Occidentales entre Wałbrzych et Bolków (Szczawno, Rochowice St.) et les pentes du nord des Monts d'Izera. Dans le bloc précédent des Sudètes on a aussi trouvé de l'eau carbogazeuse, à forte teneur en $\mathrm{CO}_{2}$ à une profondeur d'environ $500 \mathrm{~m}$.

La composition chimique des eaux carbogazeuses est déterminée pour une grande part par le type des roches apparaissant sur un terrain donné. On peut citer ici comme exemple les eaux de Polanica circulant dans les couches du Crétacé supérieur riches en calcite; le cation prédominant dans ces eaux est le calcium. Les quantités considérables de sodium dans les eaux carbogazeuses de Szczawno semblent être liées à la présence commune des feldspaths sodiques dans les sédiments du Carbonifère inférieur, desquels ces eaux sont extraites.

Parfois les eaux carbogazeuses contiennent - en dehors du bioxyde de carbone - d'autres composantes curatives spécifiques liées à la métallisation locale (par exemple 
l'arsenic dans les eaux carbogazeuses de Kudowa) ou bien à la lithologie particulière des roches et leurs déformations tectoniques (le radon dans les eaux de Świeradów).

La situation tectonique locale et les différences d'altitude entre les zones d'alimentation et de drainage mènent parfois à la formation des eaux thermales peu minéralisées, qui jaillissent sous forme de sources. Elles contiennent des composants spécifiques comme HS, F, Rn (Lądek) ou bien $\mathrm{H}_{2} \mathrm{SiO}_{3}$ (Cieplice).

\section{LA BASSE-PLAINE}

Exception faite des territoires limités comme la partie Nord-Est du pays, dans toute la Basse-Plaine existent les eaux chlorurées-sodiques contenant des sels dissous à des concentrations de quelques $\mathrm{g} / \mathrm{l}$ à quelques centaines de $\mathrm{g} / \mathrm{l}$. Elles apparaissent localement à la surface comme des sources salées (Kołobrzeg, Ciechocinek) qui ont été longtemps exploitées pour sauner le sel. Dans les couches profondes du Jurassique, Triassique et, bien sûr du Paléozoïque, la présence d'eaux salées est commune dans toute la région. L'origine de la salinité est l'objet de discussion. Certes, la présence de couches épaisses de sel qui se sont formées au Permien supérieur (Zechstein) est un facteur important pour la composition des eaux dans les formations superposées ainsi que sous-jacentes. Plusieurs diapirs des sels de Zechstein perchent les formations du Mésozoïque et parfois même du Kénozoïque, ce qui facilite le lessivage du sel par les eaux souterraines. De l'autre côté, une contribution des eaux connées des bassins marins du Mésozö̈que n'est pas exclue, selon les résultats des recherches isotopiques. Finalement, les eaux-mères sursaturées étant le résidu du bassin évaporitique de Zechstein, pourraient aussi être un des facteurs de la salinisation.

Les eaux chlorurées sodiques sont captées dans la région de la Basse-Plaine dans les couches du Mésozoïque, sporadiquement dans le Quaternaire (Kołobrzeg, Inowrocław). La profondeur des forages varie en général entre quelques dizaines de mètres et quelques centaines de mètres. Parfois, cependant elle dépasse considérablement $1000 \mathrm{~m}$ (Konstancin, Ciechocinek, Koszuty, Połczyn) surtout dans les lieux où les eaux minérales extraites sont en même temps thermales.

Parmi les composants spécifiques de ces eaux, le brome et l'iode sont fréquents. Le contenu du brome arrive parfois jusqu’à $300 \mathrm{mg} / \mathrm{l}$ (Połczyn). Dans les eaux chlorurées sodiques thermales, on observe la présence d'hydrogène sulfuré. Son origine n'a pas encore été expliquée.

Les eaux à hydrosulfures, sulfatées-chloruréescalciques-sodiques de Wieniec sont liées à une série gypsifère locale dans le Jurassique supérieur. La réduction du gypse a lieu en présence de la matière organique des lignites du Miocène.

Les eaux ferrugineuses, peu minéralisées, sont bien répandues dans le Quaternaire et le Crétacé de la BassePlaine. Des eaux de ce type ont été déclarées médicinales à Nałęczów, près de Lublin.

Outre l'application des eaux minérales, eaux oligominérales à composantes curatives spécifiques et eaux thermales comme moyens curatifs du thermalisme, les eaux thermales sont de plus en plus utilisées pour le chauffage. Des projets bien avancés de chauffage géothermique des villes Nowy Targ (Carpates) et Pyrzyce (Basse-Plaine, près de Szczecin) sont à mentionner. Parmi les avantages de l'utilisation de l'énergie géothermique de cette manière, la protection du milieu naturel contre les produits de combustion du charbon est le plus important.

\section{Bibliographie succincte}

Dowgiatlo J. (1994). - The hydrogeothermal potential of Poland. Dans : Risler J. J., Simmers J. (Rédacteurs) : Hydrogeothermics. International Contributions to Hydrogeology, Vol. 15. Ed. Heise, Hannover.

Dowgiallo J., PlochnieWSKI Z., SZPAKIEWICZ M. (1974). Map of Mineral Waters of Poland. 1 :1 500000 Warszawa.

Malınowski J. (Red.) (1991). Budowa geologiczna Polski (La structure géologique de Pologne), Vol VII. Hydrogeologia. Wyd. Geologiczne. Warszawa.

Szmytówna M. (Red.) (1970), Balneochemia. Warszawa. 